\title{
Improvement of Atrial Signal-Averaged Electrocardiographic Abnormalities After Radiofrequency Catheter Ablation in Persistent Atrial Flutter
}

\author{
Fumiharu MiUra, ${ }^{1} \mathrm{MD}$, Hidekazu HIRAO,${ }^{1} \mathrm{MD}$, Yukiko NAKANO,${ }^{1} \mathrm{MD}$, \\ Hiroki Teragawa, ${ }^{1}$ MD, Tetsuji Shingu, ${ }^{1}$ MD, and Kazuaki ChayAma, ${ }^{1}$ MD
}

\begin{abstract}
SUMMARY
It has been reported that abnormalities of atrial conduction are present in patients with atrial flutter (AFL). We analyzed the $\mathrm{P}$ wave signal-averaged ECG (PSE) in patients after cardioversion of chronic AFL by radiofrequency catheter ablation (RFCA) to determine whether abnormalities of atrial conduction exist in patients with AFL and whether they recover. We studied 11 patients undergoing ablation of persistent AFL (AFL group), 11 patients with paroxysmal AFL (PAFL group), and 14 patients without any evidence of arrhythmias (control group). The PSEs were recorded 1 day, 7 days, and 1 month after RFCA. The filtered P wave duration (FPD) was calculated from the PSE recording. The FPD correlated with interatrial conduction time $(\mathrm{r}=0.644)$ and left atrial dimension $(\mathrm{r}=$ $0.675)$ in combined assessment of the AFL and PAFL groups. The FPD was longer in the AFL group 1 day $(165 \pm 14 \mathrm{~ms}, P<0.001)$ and 1 month $(150 \pm 18 \mathrm{~ms}, P<0.05)$ after RFCA than in the control group (134 $\pm 10 \mathrm{~ms}$ ). Our findings suggest that atrial conduction abnormalities detected by PSE are present in patients with persistent AFL and improve 1 month after cardioversion. (Jpn Heart J 2004; 45: 761-770)
\end{abstract}

Key words: Atrial flutter, Interatrial conduction abnormalities, $\mathrm{P}$ wave signal-averaged ECG, Filtered $\mathrm{P}$ wave duration, Radiofrequency catheter ablation

IT has been suggested that atrial flutter (AFL) produces electrical remodeling, producing the substrate for sustained AFL in an animal model. ${ }^{1)}$ Several studies have reported electrical remodeling of the atrium in the setting of chronic AFL in humans. ${ }^{2-4)}$ Franz, et al reported that chronic AFL also may produce electrical remodeling similar to that observed with atrial fibrillation (AF) with respect to the refractory period. ${ }^{3)}$ Sparks, et al reported that atrial refractoriness increases late after termination of AFL by radiofrequency catheter ablation (RFCA) in

From the ${ }^{1}$ Department of Medicine and Molecular Science, Division of Frontier Medical Science, Programs for Biomedical Research, Graduate School of Biomedical Sciences, Hiroshima University, Hiroshima, Japan.

Address for correspondence: Fumiharu Miura, MD, Department of Medicine and Molecular Science, Division of Frontier Medical Science, Programs for Biomedical Research, Graduate School of Biomedical Sciences, Hiroshima University, 1-2-3 Kasumi, Minami-ku, Hiroshima 734-8551, Japan.

Received for publication December 8, 2003.

Revised and accepted March 1, 2004. 
patients with chronic AFL compared to the atrial refractoriness just after RFCA. ${ }^{2)}$ Based on these findings, it has been suggested that persistent AFL promotes reversible remodeling of the atrial electrical properties.

The correlation between the presence of atrial conduction abnormalities and the initiation of paroxysmal atrial fibrillation (AF) has been well documented. ${ }^{5-8)}$ It has also been reported that prolonged, fractionated atrial endocardial electrograms are frequently observed in patients with paroxysmal AF during sinus rhythm. ${ }^{9)}$ The $\mathrm{P}$ wave signal-averaged electrocardiogram (PSE) has commonly been used in patients with atrial arrhythmias. Using PSE, we can noninvasively detect abnormalities of atrial conduction. A prolonged $\mathrm{P}$ wave measured by the PSE is a useful predictor of paroxysmal AF. Therefore, we should be able to identify patients at risk for paroxysmal AF using the PSE. ${ }^{5)}$ However, the PSE in patients with persistent AFL has not been determined.

This study was performed to examine the PSE in patients with successful RFCA of chronic AFL. Our aim was to evaluate whether patients with AFL have abnormalities in the PSE, and if abnormalities existed, whether they were reversible.

\section{Methods}

Patient selection: The study population consisted of three groups: an AFL group, paroxysmal AFL (PAFL) group, and control group. The AFL group included 11 patients (10 men, 1 woman; $65 \pm 12$ years of age, range: 37 to 77 years) who underwent successful RFCA of persistent typical AFL. Persistent typical AFL was defined by the presence of AFL on the ECG on more than 2 separate occasions over a 1 month period before the ablation. The other patients had no evidence of organic heart disease. Antiarrhythmic drugs, including calcium channel blockers and digitalis, were stopped for more than 5 half-lives before the ablation. Four patients had a history of hypertension, and 3 of these patients continued to take only ACE inhibitors after the ablation. Four patients continued to take anticoagulant drugs. Eight patients continued to take antiplatelet drugs.

The PAFL group consisted of 11 patients (11 men, $68 \pm 8$ years of age, range: 37 to 77 years) who underwent successful tricuspid annulus - inferior vena cava isthmus (TA-IVC) linear ablation. Three patients had a history of hypertension, but no patients had evidence of organic heart disease. Two of these patients took ACE inhibitors.

The control group consisted of 14 age-matched patients with normal ECGs and without any tachyarrhythmias (13 men, 1 woman; $61 \pm 9$ years of age, range: 40 to 78 years). No patients had episodes of AF or any documented AF or AFL 
on standard ECG or 24-hour Holter monitoring. Four patients had a history of hypertension, 2 of whom took ACE inhibitors.

Written informed consent was obtained from all patients prior to the study. The protocol was approved by the ethics committee of our institution.

Electrophysiological study and radiofrequency catheter ablation: In the AFL and PAFL groups, intracardiac multipolar electrode catheters were placed in the high right atrium, coronary sinus, His bundle, and tricuspid annulus positions. The ablation catheter was positioned at the TA-IVC for entrainment mapping and RFCA in the AFL group. The AFL was induced by programmed stimulation and the AFL mechanism was confirmed by entrainment of the TA-IVC isthmus in the PAFL group. After confirmation of the AFL mechanism, RFCA was performed by creating a linear lesion between the TA and IVC. In all patients, the ablation was performed during AFL. The endpoint of the AFL ablation was the formation of complete bidirectional isthmus block. After the ablation, AF could not be induced by programmed stimulation.

PSE recordings: The PSE was recorded with a multipurpose electrocardiograph (VCM-3000, Fukuda Denshi Co., Tokyo) in an electrically shielded room. The patients were placed in the supine position and were asked to relax during the recording. The skin was cleaned with alcohol. Three electrode pairs were placed in the following locations: The $\mathrm{X}$ lead was positioned between the right and left shoulders, and represented standard lead I. The aVF lead was used as the Y lead, and the precordial $\mathrm{V}_{1}$ lead as the $\mathrm{Z}$ lead. The signal from each lead was amplified to $5 \mu \mathrm{V} / \mathrm{cm}$, and passed through a $40-\mathrm{Hz}$ high-pass filter (slope: $18 \mathrm{~dB} / \mathrm{oct}$ ) and 300-Hz low-pass filter (slope: $12 \mathrm{~dB} / \mathrm{oct}$ ). We also used a forward filter. The PSE was measured using an automatic template recognition algorithm for the P-wave triggering system. $\mathrm{P}$ wave triggering signals for 500 beats during sinus rhythm were integrated. After the 500 beat integration, the noise level was $<0.5 \mu \mathrm{V}$. In the control group, the PSE was recorded in the same fashion as that in the AFL group.

Data analysis: The $\mathrm{P}$ wave complexes from the three bipolar leads were combined into a vector magnitude using the formula $\sqrt{\mathrm{X}^{2}+\mathrm{Y}^{2}+\mathrm{Z}^{2}}$. The onset and offset of the $\mathrm{P}$ waves were defined as the points where the voltage of the atrial signal exceeded $0.5 \mu \mathrm{V}$ during the TP segment and returned to a level of $0.5 \mu \mathrm{V}$, respectively. The filtered $\mathrm{P}$ wave duration (FPD) was measured in all individuals.

Study protocol: In the AFL group, the PSE was recorded 1 day (day 1), 7 days (day 7), and 1 month (1M) after the ablation. After the ablation, all patients were followed-up every 2 weeks for 1 month to obtain a 12-lead ECG recording and underwent a physical examination. If a patient had palpitations suggestive of a tachycardia, a Holter ECG was obtained. Even when a patient had no palpitations, a Holter ECG was obtained 1 month after the ablation. In the PAFL group, 
the PSE was recorded 1 day before the ablation and 1 day after the ablation to determine whether the RFCA influenced the value of the PSE. In the control group, the PSE were recorded twice over a 1 month interval.

Statistical analysis: All continuous variables are expressed as the mean $\pm \mathrm{SD}$. A repeated measure ANOVA was used to compare the continuous variables in the AFL group. A two-tailed paired or unpaired Student's $t$ test was performed when appropriate. Pearson's correlation coefficient was used to compare the PSE parameters with other parameters. Statistical significance was established at a value of $P<0.05$.

\section{RESULTS}

Patient characteristics: The patient characteristics are summarized in Table I. Age, gender, left ventricular ejection fraction, and the frequencies of hypertension and atrial septal defect repair were similar among the three groups. The patients in the AFL group had persistent AFL for $21 \pm 12$ months (range: 1 to 136 months). The flutter cycle length (FCL) was $247 \pm 22 \mathrm{~ms}$ (range: 208 to $255 \mathrm{~ms}$ ). The left atrial dimension (LAD) in the AFL group was significantly larger than in the control group (39 \pm 4 versus $35 \pm 4 \mathrm{~mm}, P<0.05$ ). The LAD in the PAFL group was similar to that in the control group. The FCL in the PAFL group was $244 \pm 21 \mathrm{~ms}$ (range: 202 to $280 \mathrm{~ms}$ ). The FCL did not differ between the AFL group and the PAFL group.

Follow-up data: No patients experienced a recurrence of the AFL during the 1 month following ablation. Two patients had documented paroxysmal AF within 1 month (8 days and 10 days, respectively, after the ablation), which was sustained for 12 hours and 26 hours, respectively. In both patients, the AF spontaneously terminated and no antiarrhythmic drugs were needed.

Table I. Clinical Characteristics of the Patients

\begin{tabular}{lcccc}
\hline & AFL group & PAFL group & Control group & $P$ value \\
\hline Number of cases & 11 & 11 & 14 & NS \\
Age (years) & $65 \pm 12$ & $68 \pm 8$ & $61 \pm 9$ & NS \\
Gender (male/female) & $10 / 1$ & $11 / 0$ & $13 / 1$ & NS \\
LAD (mm) & $* 39 \pm 4$ & $* 37 \pm 4$ & $35 \pm 4$ & $<0.05$ \\
LVEF (\%) & $67.8 \pm 5.6$ & $68.8 \pm 8.0$ & $68.7 \pm 9.5$ & NS \\
Hypertension & 4 & 3 & 4 & NS \\
Duration of AFL (months) & $21 \pm 12$ & - & - & \\
& $(1-136)$ & & & NS \\
FCL (ms) & $247 \pm 22$ & $244 \pm 21$ & - & \\
\hline
\end{tabular}

Data are presented as the mean $\pm \mathrm{SD} . * P<0.05$ versus Control. LAD $=$ left atrial dimension; $\mathrm{LVEF}=$ left ventricular ejection fraction; FCL = flutter cycle length; $\mathrm{AFL}=$ atrial flutter; $\mathrm{PAFL}=$ paroxysmal atrial flutter. 
Correlation between the PSE and other parameters: In a combined assessment of the AFL and PAFL groups, the FPD on day 1 correlated positively with LAD $(0.675, P=0.0005)$ and with interatrial conduction time (interval from onset of the $\mathrm{P}$ wave to onset of the atrial potential in the distal coronary sinus, $\mathrm{r}=0.644, P$ $=0.0012$, Figure 1$)$. The FPD did not correlate with age, history of hypertension, gender, or left ventricular ejection fraction.

Acute effect of RFCA on FPD and LAD: The FPD was significantly longer in the PAFL group before the ablation than the control group (140 \pm 15 versus $134 \pm 10$ ms, $P<0.05$, Table II). The FPD did not change after the ablation in the PAFL group (Table II, Figure 2). The LAD did not change in either the AFL or PAFL groups after ablation.

Changes in FPD and LAD: Typical changes in PSE in the AFL group are illustrated in Figure 3. The FPD on day 1 in the AFL group was significantly longer than in the control group ( $165 \pm 14$ versus $134 \pm 10 \mathrm{~ms}, P<0.0001$, Table II, Figure 4$)$. The FPD gradually shortened after cardioversion $(P<0.05)$. The FPD on
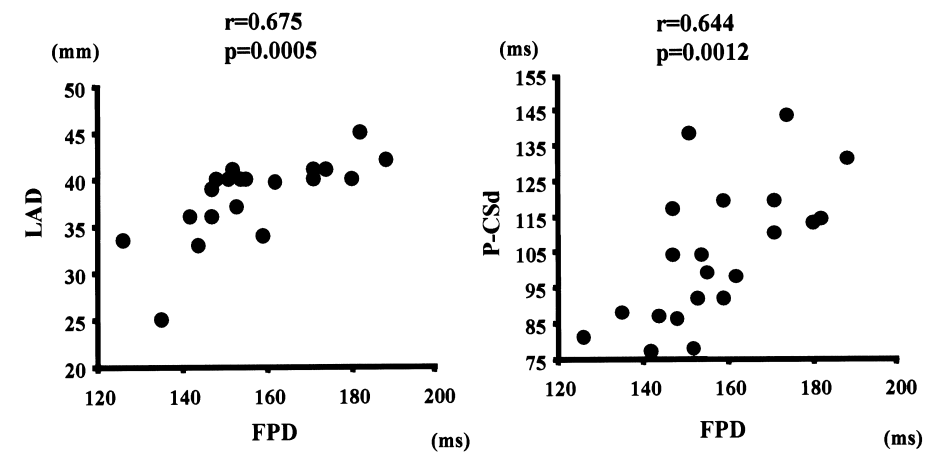

Figure 1. Relationship between the FPD and LA size (left), and interatrial conduction time (right). $\mathrm{FPD}=$ filtered $P$ wave duration; $\mathrm{LA}=$ left atrial $\mathrm{LAD}=$ left atrial dimension $; \mathrm{P}-\mathrm{CSd}=$ conduction time from the onset of the $P$ wave on the surface ECG to the onset of the atrial potential in the distal coronary sinus.

Table II. Changes in the Filtered $P$ Wave Duration and Left Atrial Dimension in the Control, PAFL, and AFL groups

\begin{tabular}{lcccccc}
\hline & \multirow{2}{*}{$\begin{array}{c}\text { Control } \\
\text { group }\end{array}$} & Pre RFCA & Post RFCA & 1 day after & 7 days after & 1 month after \\
& & & & & & \\
& & & & & \\
& & & & \\
FPD $(\mathrm{ms})$ & $134 \pm 10$ & $140 \pm 15^{*}$ & $144 \pm 15^{*}$ & $165 \pm 14^{*}$ & $164 \pm 19^{*}$ & $150 \pm 18^{\# *}$ \\
LAD $(\mathrm{mm})$ & $35 \pm 4$ & $37 \pm 4$ & $37 \pm 5$ & $39 \pm 4^{*}$ & - & $38 \pm 3^{*}$ \\
\hline
\end{tabular}

Data are presented as the mean \pm SD. ${ }^{*} P<0.05$ versus control group. ${ }^{*} P<0.05$ versus 1 day after the ablation in the AFL group. PSE = P wave signal-averaged ECG; AFL = atrial flutter; FPD = filtered $\mathrm{P}$ wave duration; RFCA=radiofrequency catheter ablation; $\mathrm{LAD}=$ left atrial dimension. 


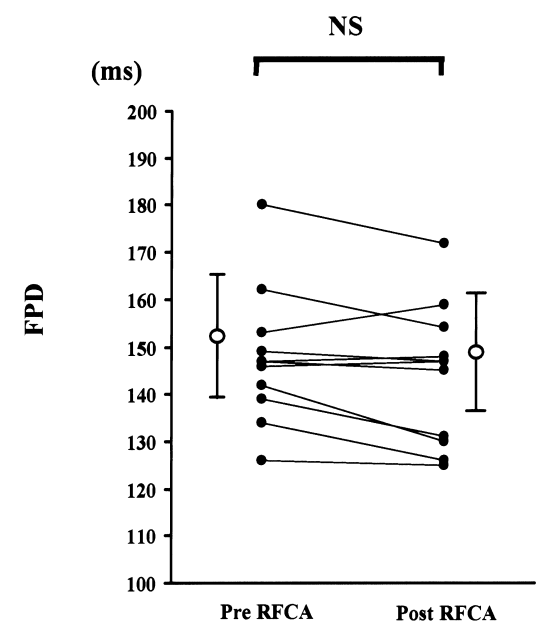

Figure 2. Acute changes in the FPD just after the RFCA in the PAFL group.

PAFL $=$ paroxysmal atrial flutter; RFCA $=$ radiofrequency catheter ablation; $\mathrm{FPD}=$ filtered $\mathrm{P}$ wave duration.

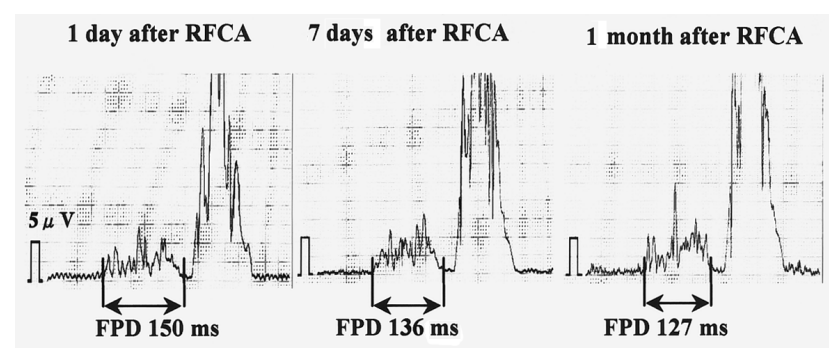

Figure 3. $\mathrm{P}$ wave signal-averaged ECG in a representative case in the AFL group. This patient was a 63 year-old man. The duration of the AFL was 10 months. The flutter cycle length was $230 \mathrm{~ms}$. This figure shows the PSE on day 1, after 7 days, and 1 month after the ablation. The prolonged FPD 1 day after cardioversion gradually shortened after cardioversion. $\mathrm{AFL}=$ atrial flutter; $\mathrm{PSE}=\mathrm{P}$ wave signal-averaged $\mathrm{ECG} ; \mathrm{RFCA}=$ radiofrequency catheter ablation; $\mathrm{FPD}=$ filtered $\mathrm{P}$ wave duration.

day 7 was similar to that on day 1 . However, the FPD after 1 month was significantly shorter than that on day $1(150 \pm 18$ versus $165 \pm 14 \mathrm{~ms}, P<0.05)$. The FPD after 1 month was still significantly longer than in the control group (150 \pm 18 versus $134 \pm 10 \mathrm{~ms}, P<0.005)$. The LAD after 1 month was not significantly different from that on day 1. 


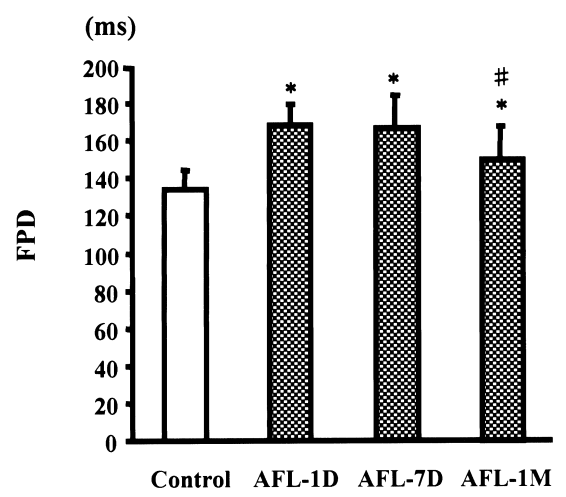

Figure 4. The time course for changes in the FPD in the AFL groups. The open bar represents the control group, and the solid bars represent the AFL group. ${ }^{*} P<0.05$ versus control. ${ }^{\#} P<0.05$ versus 1 day after cardioversion in the AFL group. $\mathrm{FPD}=$ filtered $\mathrm{P}$ wave duration; $\mathrm{AFL}=$ atrial flutter; AFL-1D = 1 day after cardioversion; AFL$7 \mathrm{D}=7$ days after cardioversion; AFL-1M $=1$ month after cardioversion.

\section{DISCUSSION}

The present study produced the following main findings: 1) The FPD correlates with interatrial conduction time, 2) the FPD is longer in patients with AFL than in normal individuals, and 3) the PSE in patients with AFL improves 1 month after cardioversion. These findings suggest that atrial conduction abnormalities, assessed using FPD, are present in patients with persistent AFL and improve within 1 month after cardioversion. PSE recordings may be useful in detecting atrial conduction abnormalities noninvasively in such patients.

Previous studies have reported that the FPD correlates with LAD, age, and history of hypertension. ${ }^{11-15)}$ It has been reported that the initial potentials correlate with indices of electrophysiologic measurements, although the statistical significance is weak. ${ }^{16)}$ In the present study, we demonstrated that the FPD correlates positively with interatrial conduction time, which is one of the electrophysiologic indices for atrial conduction abnormalities. This finding suggests that atrial conduction abnormalities may be detected noninvasively using the PSE.

Fukunami, et al reported that the FPD is longer in patients with paroxysmal AF than in those without. ${ }^{6)}$ However, the FPD in patients with chronic AFL has not been reported. The present study showed that the FPD is longer in patients with persistent AFL just after ablation. This suggests that patients with persistent AFL have conduction abnormalities during rapid atrial activation. Because the 
FPD before and after the RFCA in the PAFL group was similar, there is little possibility that the RFCA affects the FPD measurement.

Previous electrophysiologic studies have reported that slowly conducting atrial activation and atrial late potentials are present in paroxysmal AF. ${ }^{9,10,16)}$ Buxton, et al reported in patients with spontaneous AFL that there is a delay in the conduction of early premature atrial stimuli. ${ }^{9)}$ Further, another study reported that AFL is associated with a prolongation of the right intra atrial conduction time and mean interatrial conduction time. ${ }^{10)}$ These findings suggest that atrial conduction abnormalities are present in patients with AFL. Our observations using the PSE are consistent with the results reported in the previous electrophysiologic studies. $^{9,10)}$

Recently, there have been several reports about atrial remodeling due to AFL. ${ }^{2-4)}$ Sparks, et al demonstrated that in the setting of chronic AFL, the atrial effective refractory period increases 3 weeks after the ablation. ${ }^{2)}$ Franz, et al reported that there is a quantitatively similar decrease in the monophasic action potential recording at $90 \%$ depolarization for both patients with AF or AFL. ${ }^{3)}$ Further, in another report, temporal recovery of the corrected sinus node recovery time after the ablation of chronic AFL has been reported. ${ }^{4)}$ Previous studies indicate that the change in the atrial electrical properties is reversible after cardioversion of AFL. ${ }^{2-4)}$ In animal models, chronic rapid atrial activation provides abnormalities in atrial conduction and causes electrical remodeling that promotes AF. ${ }^{17)}$ However, no studies that considered the changes in atrial conduction abnormalities have been reported in humans. We considered the atrial conduction time, based on the PSE, to be an index of the atrial electrical properties, because it correlates with the interatrial conduction time. In the present study, the FPD became shorter 1 month after the ablation compared with just after the ablation in the AFL group, but it was still longer than that in the control group. These results suggest that atrial conduction abnormalities in the AFL are reversible and may improve to some extent within 1 month after cardioversion.

In the present study, abnormalities of atrial conduction improved 1 month after the RFCA although LAD did not decrease in this period. A previous study ${ }^{18}$ has reported that left atrial appendage contraction improved 2 weeks after RFCA for chronic AFL. We hypothesize that the improvement in atrial conduction occurs first, followed by structural remodeling.

In some patients, paroxysmal AF occurs after successful ablation of AFL. In this study, paroxysmal AF occurred transiently in 2 patients 8 and 10 days, respectively, after the RFCA for AFL. Since the number of patients is small we can not discuss statistical differences in PSE. However, the PSE in these two patients was similar to the other patients. Previous follow-up studies also showed that AF can occur after AFL ablation, but that it is generally transient, and never 
requires long-term antiarrhythmic therapy. ${ }^{19-21)}$ It has also been reported that the ablation of AFL in patients who have both AFL and AF is associated with a reduced incidence of subsequent AF of approximately 50\%. This phenomenon may account for the prolonged atrial conduction observed in the setting of AFL in addition to the electrical remodeling resulting in changes in the effective refractory period. It has been suggested that improving the atrial conduction abnormalities would remove the substrate responsible for the development of AF. Study limitations: Previous studies have also shown that AF can occur in patients undergoing AFL ablation. ${ }^{19-21)}$ In the present study, paroxysmal AF occurred in 2 patients after the RFCA for AFL. Because the number of patients with AF was so small, we could not evaluate the FPD or other parameters. Further studies of the occurrence of AF or the recurrence of AFL over 1 month are needed. Second, in the present study, we did not determine the FPD more than 1 month after RFCA in patients with persistent AFL. Therefore, it is unclear whether the shortening of FPD continues after more than 1 month. Third, we examined the relationship between the FPD and LAD in patients who underwent RFCA for AFL, and we found that the FPD improved without any reduction in the LAD. However, we did not examine other parameters of atrial function, which may correlate with the FPD. Lastly, the atrial conduction time abnormalities recovered incompletely and the LAD at 1 month after cardioversion was still greater than that in the control group after 1 month in the present study. This may be because the period of observation was too short.

Conculusion: The PSE is a noninvasive tool for evaluating serial changes in atrial conduction. However, further studies are needed to examine whether the PSE is useful in distinguishing patients with recurrent AFL or AF from those without recurrent AFL or AF after ablation.

\section{REFERENCES}

1. Morton JB, Byrne MJ, Power JM, Raman J, Kalman JM. Electrical remodeling of the atrium in an anatomic model of atrial flutter: relationship between substrate and triggers for conversion to atrial fibrillation. Circulation 2002; 105: 258-64.

2. Sparks PB, Jayaprakash S, Vohra JK, Kalman JM. Electrical remodeling of the atria associated with paroxysmal and chronic atrial flutter. Circulation 2000; 102: 1807-13.

3. Franz MR, Karasik PL, Li C, Moubarak J, Chavez M. Electrical remodeling of the human atrium: similar effects in patients with chronic atrial fibrillation and atrial flutter. J Am Coll Cardiol 1997; 30: 1785-92.

4. Daoud EG, Weiss R, Augostini RS, Moubarak J, Chavez M. Remodeling of sinus node function after catheter ablation of right atrial flutter. J Cardiovasc Electrophysiol 2002; 13: 20-4.

5. Engel TR, Vallone N, Windle J. Signal-averaged electrocardiograms in patients with atrial fibrillation or flutter. Am Heart J 1988; 115: 592-7.

6. Fukunami M, Yamada T, Ohmori M, et al. Detection of patients at risk for paroxysmal atrial fibrillation during sinus rhythm by P wave-triggered signal-averaged electrocardiogram. Circulation 1991; 83: 162-9. 
7. Guidera SA, Steinberg JS. The signal-averaged $\mathrm{P}$ wave duration: a rapid and noninvasive marker of risk of atrial fibrillation. J Am Coll Cardiol 1993; 21: 1645-51.

8. Darbar D, Jahangir A, Hammill SC, Gersh BJ. P wave signal-averaged electrocardiography to identify risk for atrial fibrillation. Pacing Clin Electrophysiol 2002; 25: 1447-53. (Review)

9. Buxton AE, Waxman HL, Marchlinski FE, Josephson ME. Atrial conduction: effects of extrastimuli with and without atrial dysrhythmias. Am J Cardiol 1984; 54: 755-61.

10. Leier CV, Meacham JA, Schaal SF. Prolonged atrial conduction. A major predisposing factor for the development of atrial flutter. Circulation 1978; 57: 213-6.

11. Madu EC, Baugh DS, Gbadebo TD, Dhala A, Cardoso S; Phi-Res Multi Study Group. Effect of ethnicity and hypertension on atrial conduction: evaluation with high-resolution P-wave signal averaging. Clin Cardiol 2001; 24: 597-602.

12. Ogawa H, Inoue $\mathrm{T}$, Yoshida A, et al. The signal-averaged electrocardiogram of $\mathrm{P}$ wave in patients with documented atrial fibrillation or flutter and in patients with left or right atrial overload without atrial fibrillation. Jpn Heart J 1993; 34: 29-39.

13. Ehrlich JR, Steul K, Schadow K, Breuer S, Hohnloser SH. Relationship between clinical and echocardiography-derived parameters and atrial activation as measured by the $\mathrm{P}$-wave signal-averaged electrocardiogram. Z Kardiol 2002; 91: 404-9.

14. Babaev AA, Vloka ME, Sadurski R, Steinberg JS. Influence of age on atrial activation as measured by the Pwave signal-averaged electrocardiogram. Am J Cardiol 2000; 86: 692-5, A9.

15. Furihata A, Ozawa Y, Kasamaki Y, Watanabe I, Yanagawa S, Saito S. Age and sex differences in the P-wave signal-averaged electrocardiogram in a Japanese study population. Jpn Heart J 2001; 42: 295-305.

16. Sasaki R, Sugisawa K, Tani H, et al. Correlation between initial potentials on a signal-averaged P-wave and indice of electrophysiologic measurements in the right atrium. Jpn Circ J 1998; 62: 279-83.

17. Gaspo R, Bosch RF, Talajic M, Nattel S. Functional mechanisms underlying tachycardia - induced sustained atrial fibrillation in a chronic dog model. Circulation 1997; 96: 4027-35.

18. Takami M, Suzuki M, Suga K, Ikeda T. Time course for resolution of left atrial appendage stunning after catheter ablation of chronic atrial flutter. J Am Coll Cardiol 2003; 41: 2207-11.

19. Philippon F, Plumb VJ, Epstein AE, Kay GN. The risk of atrial fibrillation following radiofrequency catheter ablation of atrial flutter. Circulation 1995; 92: 430-5.

20. Movsowitz C, Callans DJ, Schwartzman D, Gottlieb C, Marchlinski FE. The results of atrial flutter ablation in patients with and without a history of atrial fibrillation. Am J Cardiol 1996; 78: 93-6.

21. Loutrianakis E, Barakat T, Olshansky B. Early versus late atrial fibrillation after atrial flutter ablation. J Interv Card Electrophysiol 2002; 6: 173-80. 